\title{
Fight against Covid-19 from the Perspective of Deaths
}

\author{
Angela Luciana De-Bortoli \\ Business Partner - Entrepreneur \\ Aracaju, Brasil \\ Lia Breda-Vicentini \\ Psychology College \\ Paulista University - UNIP \\ Sorocaba, Brasil \\ Robelius De-Bortoli \\ Federal University of Sergipe \\ Aracaju, Brasil
}

\begin{abstract}
On March 11, the World Health Organization declared the state of the global pandemic for COVID-19. Efforts are focused on blocking the evolution of the disease while preventive measures (vaccines) or prophylactics (medication for treatment) are not known, accepted or validated by the scientific community. In a very conscious way, the measures are focused on reducing the transmission of SARS-CoV-2 (Severe Acute Respiratory Syndrome Coronavirus 2), believing that this will reduce the number of deaths. Based on this evidence presented in the Brazilian case, it is possible to state that deaths related to COVID-19 are concentrated in older populations and with associated comorbidities or chronic diseases. The lockdown system is more efficient to isolate populations with higher mortality (age and comorbidity). Younger populations are less affected by COVID-19, making them good agents of transmission. The challenge is to access the population that is dying before COVID-19 is in an advanced stage so that there is more and better time to fight the disease.
\end{abstract}

Keywords: COVID-19, Severe Acute Respiratory Syndrome Coronavirus, Health Policy

The year 2020 will be remembered for being a year in which the world felt the effects of a Pandemic with global effects (Accinelli et al, 2020). All actions proposed by the bodies linked to health care suffer and will suffer political bias (Araújo, Oliveira and Freitas, 2020), as their consequences impact on the countries' economic activities. When a researcher manifests himself, his words must be interpreted as thoughts for future actions, as all possible effects are delayed, not interacting with past events. Scientific interpretation cannot be seen as manifest, but as a way of visualizing a problem, enabling the multiplication of options for similar events. It could also be an aid to understanding the past to contribute to the improvement of the human future.

This year will be marked by the fight against COVID-19, defined as a death resulting from a clinically compatible disease in a probable or confirmed case of COVID-19, unless there is a clear alternative cause of death that cannot be related to COVID-19 disease. There should be no full recovery period between illness and death (WHO, 2020a).

This disease took on a worldwide profile due to the rapid and uncontrolled evolution of the number of cases. On March 11, the World Health Organization declared the state of the global pandemic. No world region has been spared. To date, the largest number of cases has been reported in Asia, Europe and North America (WHO, 2020b), but countries in the southern hemisphere such as Brazil are experiencing an increase in the number of cases at very high values.

\section{Facing the pandemic}

Efforts are focused on blocking the evolution of the disease while preventive measures (vaccines) or prophylactics (medication for treatment) are not known, accepted or validated by the scientific community. In a very conscious way, the measures are focused on reducing the transmission of SARS-CoV-2 (Severe Acute Respiratory Syndrome Coronavirus 2), believing that this will reduce the number of deaths. Among current interventions, including extended vacations and self-quarantine at home, there is clear direction to isolate high-risk populations.

The scenario at the beginning of the pandemic suggested that the mandatory isolation of Wuhan, a site accepted as the center for the spread of SARS-CoV-2, and your first level response had played a large role in stopping the spread of epidemics (Pan et al, 2020). 
New procedures and their results motivated measures such as lockdown and social isolation. The blockade in Wuhan combined with nationwide traffic restrictions and self-isolation measures was found to reduce the continued spread of COVID-19 in mainland China (Yuan et al, 2020). However, these suggested isolation procedures can cause undesirable effects on the population's health. Some studies indicate that some public health interventions designed to interrupt the transmission of COVID-19 could have deleterious impacts on access to primary health care (Siedner et al, 2020).

The countries that suffered the greatest impact initially from the COVID-19 cases presented the first strategies for combating them. Lockdown started to be implemented more and more in Europe, with the whole country of Italy in blockade. However, contrary to the strict measures implemented in Wuhan / Hubei, the Italian authorities allowed residents to continue working, in addition to eating out until $6 \mathrm{pm}$, if they respected 1 meter away from other people (Lau et al, 2020).

A study carried out in South Africa sought to identify whether the implementation of the national blockade order (shelter on site) affected the visitation of clinics in rural Kwa-Zulu Natal - KZN (Pan et al, 2020). Among its conclusions are suggestions for future studies aimed at exploring efforts to decentralize chronic care to high-risk populations.

These suggestions focused on attention to comorbidities or chronic diseases present in the population now with an indication of reduced displacement or social isolation reinforces the concern that special attention should be given to cardiovascular protection during treatment for COVID-19 (Zheng, Ma, Zhang and Xie, 2020), considering one of the chronic diseases with the highest risk of death. All this is aggravated by the difficulty of access between the Basic Health Unit and the population now oriented to stay at home.

\section{Brazilian Case}

Using the situation in Brazil as an example and considering it as an important scenario due to its territorial extension and the number of COVID-19 cases and resulting deaths, it is observed that the data of June 27 point to a total of $1,313,667$ people with results positive for COVID-19. Of these, 57,070 deaths are officially recorded ${ }^{10}$. Evidence must be observed, as among deaths by COVID-19, are male (57.4\%) and the age group with the highest number of reported deaths is 70 to 79 years old, with 20,428 ( $24.1 \%$ ) deaths.

When dividing the number of deaths by the population of its specific age group, the ratio of deaths per 100,000 inhabitants of the age group is presented with the following numbers: age range from 0 to 19 years $=0.2 ; 20$ to 29 years $=1.8 ; 30$ to 39 years $=6.8 ; 40$ to 49 years $=16.9 ; 50$ to 59 years $=43.1 ; 60$ to 69 years $=112.2 ; 70$ to 79 years $=180.3$; 80 years or older $=388.6$. It is observed that in this picture, mortality is concentrated in older people. The figures indicate that COVID-19 is not a serious problem for young people (figure 1).

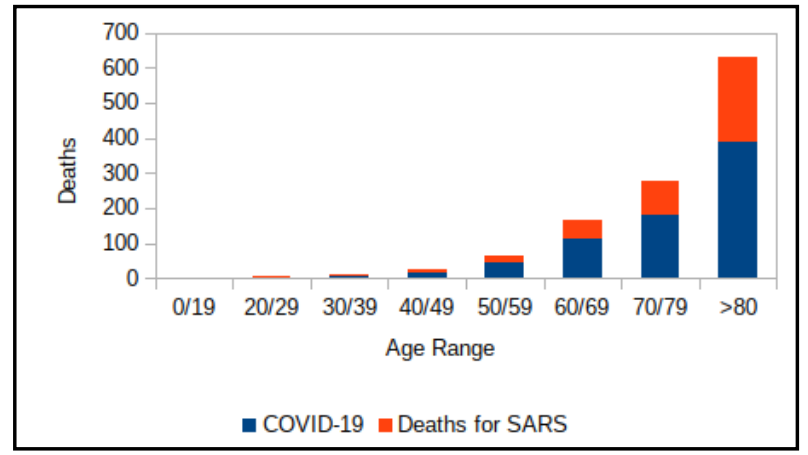

Figure 1. Total deaths by age group from COVID-19 and other SARS.

Other data also made available by the Ministry of Health of Brazil (SVA/MS, 2020) must be analyzed. They refer to comorbidities or chronic diseases present in the population that died and reported as death by COVID-19. These factors enhance the lethality of the disease. People over the age of 60 and associated comorbidity add up to the following deaths:

Heart disease, 16,958; Diabetes, 12,822; Kidney disease, 2,450 and Neurological disease, 2,660, totaling 61.1\% of the total deaths with only these 4 diseases. These numbers are equivalent to 158.4 deaths per 100,000 subjects in the age group. 


\section{Conclusions}

Based on this evidence presented in the Brazilian case, it is possible to state that deaths related to COVID-19 are concentrated in older populations and with associated comorbidities or chronic diseases. The lockdown system is more efficient to isolate populations with higher mortality (age and comorbidity). Younger populations are less affected by COVID-19, making them good agents of transmission. With guidelines for social isolation, access to the health system is impaired, especially for older populations. Finally, but no less worrisome for the records, there is no security to affirm that COVID-19 is not a consequence of the weakness installed by the pre-existing disease.

The challenge is to access the population that is dying before COVID-19 is in an advanced stage so that there is more and better time to fight the disease. We also have to define whether COVID-19 caused complications in pre-existing diseases or if the debilitation caused by pre-existing disease was the gateway to the virus and its ability to evolve.

The system for controlling the spread of the disease could not be the strongest argument for combat, but the mortality of those affected. In this sense, the isolation of the elderly and people with chronic diseases would not be justified, as they need more speed in the initial care. The basic procedures adopted in almost all countries, such as measuring body temperature, would be an important aid in the early diagnosis of COVID-19, justifying the non-isolation of the populations at greatest risk, but rather encouraging their participation in early detection actions such as taking a temperature, always respecting other procedures such as wearing a mask and interpersonal distance.

\section{Acknowledgements}

Special thanks to the work team.

Funding: The team for this work receives funding from CAPES, FAPITEC, CNPq and UFS.

Competing interests: None declared.

\section{References}

Accinelli, R. A., Xu, C. M. Z., Yachachin-Chávez, J. M., Cáceres-Pizarro, J. A., Tafur-Bances, K. B., Flores-Tejada, R. G., \& del Carmen Paiva-Andrade, A. (2020). COVID-19: La pandemia por el nuevo virus SARS-CoV-2. Revista Peruana de Medicina Experimental y Salud Pública, 37(2).

Araújo, J. L. D., Oliveira, K. K. D. D., \& Freitas, R. J. M. D. (2020). Em defesa do Sistema Único de Saúde no contexto da pandemia por SARS-CoV-2. Revista Brasileira de Enfermagem, 73.

Lau, H., Khosrawipour, V., Kocbach, P., Mikolajczyk, A., Schubert, J., Bania, J., \& Khosrawipour, T. (2020). The positive impact of lockdown in Wuhan on containing the COVID-19 outbreak in China. Journal of travel medicine, 27(3), taaa037.

Pan, J., Yao, Y., Liu, Z., Li, M., Wang, Y., Dong, W., ... \& Wang, W. (2020). Effectiveness of control strategies for Coronavirus Disease 2019: a SEIR dynamic modeling study. medRxiv.

doi: http://dx.doi.org/10.2471/BLT.20.253807

Siedner, M. J., Kraemer, J. D., Meyer, M. J., Harling, G., Mngomezulu, T., Gabela, P., ... \& Seeley, J. (2020). Access to primary healthcare during lockdown measures for COVID-19 in rural South Africa: a longitudinal cohort study. medRxiv.

SVA/MS [Secretaria de Vigilância em Saúde, Ministério da Saúde] (2020). Doença pelo Coronavírus COVID-19. Boletim Epidemiológico Especial, Semana Epidemiológica 26 (21 a 27/06). Available at: http://saude.gov.br/images/pdf/2020/July/01/Boletim-epidemiologico-COVID-20-3.pdf [Cited 2020 Jul 7].

WHO [World Health Organization, \& World health organization]. (2020a). Coronavirus disease (COVID-2019) situation reports \#167. Available at:

https://www.who.int/docs/default-source/coronaviruse/situation-reports/20200705-covid-19-sitrep167.pdf?sfvrsn=17e7e3df_4 [Cited $2020 \mathrm{Jul} 7]$.

WHO [World Health Organization, \& World health organization]. (2020b). Coronavirus disease (COVID-2019) situation reports \#51. Available at:

https://www.who.int/emergencies/diseases/novel-coronavirus-2019/situation-reports [Cited 2020 Jul 7].

Yuan, Z., Xiao, Y., Dai, Z., Huang, J., Zhang, Z., \& Chen, Y. (2020). Modelling the effects of Wuhan's lockdown during COVID-19, China. Bulletin of the World Health Organization, 98(7) doi: http://dx.doi.org/10.2471/BLT.20.254045

Zheng, Y. Y., Ma, Y. T., Zhang, J. Y., \& Xie, X. (2020). COVID-19 and the cardiovascular system. Nature Reviews Cardiology, 17(5), 259-260 doi: https://doi.org/10.1038/s41569-020-0360-5 